\title{
Design and Implementation of SOA-based Power Transmission Project Intelligent Design Review System
}

\author{
Kun Chen \\ Network planning research center \\ Guangdong Power Grid Corporation \\ Guangdong, China \\ chenkun@gd.csg.cn \\ Bochong Pan \\ Network planning research center \\ Guangdong Power Grid Corporation \\ Guangdong, China \\ panbochong@gd.csg.cn \\ Xiangbing Wang \\ Network planning research center \\ Guangdong Power Grid Corporation \\ Guangdong, China \\ wangxiangbing@gd.csg.cn
}

\author{
Guoyong Li \\ Beijing Heng Hua Albert Technology Co., Ltd. \\ Shanghai, China \\ lgy@ieforever.com \\ Zhiming Liu \\ Beijing Heng Hua Albert Technology Co., Ltd. \\ Meizhou, China \\ 1zm@ieforever.com
}

\begin{abstract}
In view of the current power transmission project design review, the requirements of automatic review, online collaborative evaluation and information intelligence analysis are increasing. This paper designed and implemented an intelligent design review system based on the idea of SOA and the SSH2 three-tier architecture. Various basic databases need to be built firstly. Then the fuzzy technology and BPR algorithm are used to vectorize the unstructured engineering reports. The system provides a comprehensive and integrated online assessment platform, which can improve the efficiency of assessment. The design reports and technical documents can be extracted and analysed intelligently, and the project key technical and economic index tables, assessment points, review draft opinions can be formed automatically, improving the quality and speed of design review. Finally, the system can analysis the engineering design and evaluation results intelligently in multi-dimensions, the engineering key chart information can be generated automatically, providing auxiliary decision basis for power grid engineering construction
\end{abstract}

Keywords-Intelligent Design Review; Auto Review; Intelligent analysis; Collaborative Review; $S O A$

\section{INTRODUCTION}

To review project design files in traditional way, artificial means is always used. The efficiency of this way is low, and the risk of mistaking and omitting review points also exists. The evaluation result data is recorded on a paper document or Excel file, and the storage locations are scattered, resulting in great workload of data collection, statistics, and analysis. So the computer and network technology is in great need to build a set of intelligent design review system, to realize informatization, standardization, streamline, intellectualization in the process of reviewing power transmission project. Not only to improve the review work efficiency, but also provides intellectual support for power grid construction.

\section{KEY TECHNOLOGY OF SYSTEM CONSTRUCTION}

The loose, coarse-grained distributed components can be arranged, mixed, and re-packaged by using the SOA architecture model, and these new components will be provided directly to the application system. Under the SOA architecture, the system can show up a variety of information resources in the form of services.

Systems based on SOA have the characteristics of loose coupling, coarse grained, transparent position and transmission protocol. The platform can meet the requirements of various components integrating flexibly and interoperability for its design of services architecture based on SOA.

The intelligent design review system is implemented by using the SSH2 three-tier architecture: Struts2, Hibernate, and Spring. Struts2 implements MVC architecture skillfully. Hibernate packaged JDBC(Java Database Connectivity Standard), thus the JAVA classes can be mapped to database tables. The Inversion of Control (IoC) design pattern is applied in Spring, and the container is responsible for the dependencies between business objects and the life cycle of objects. 


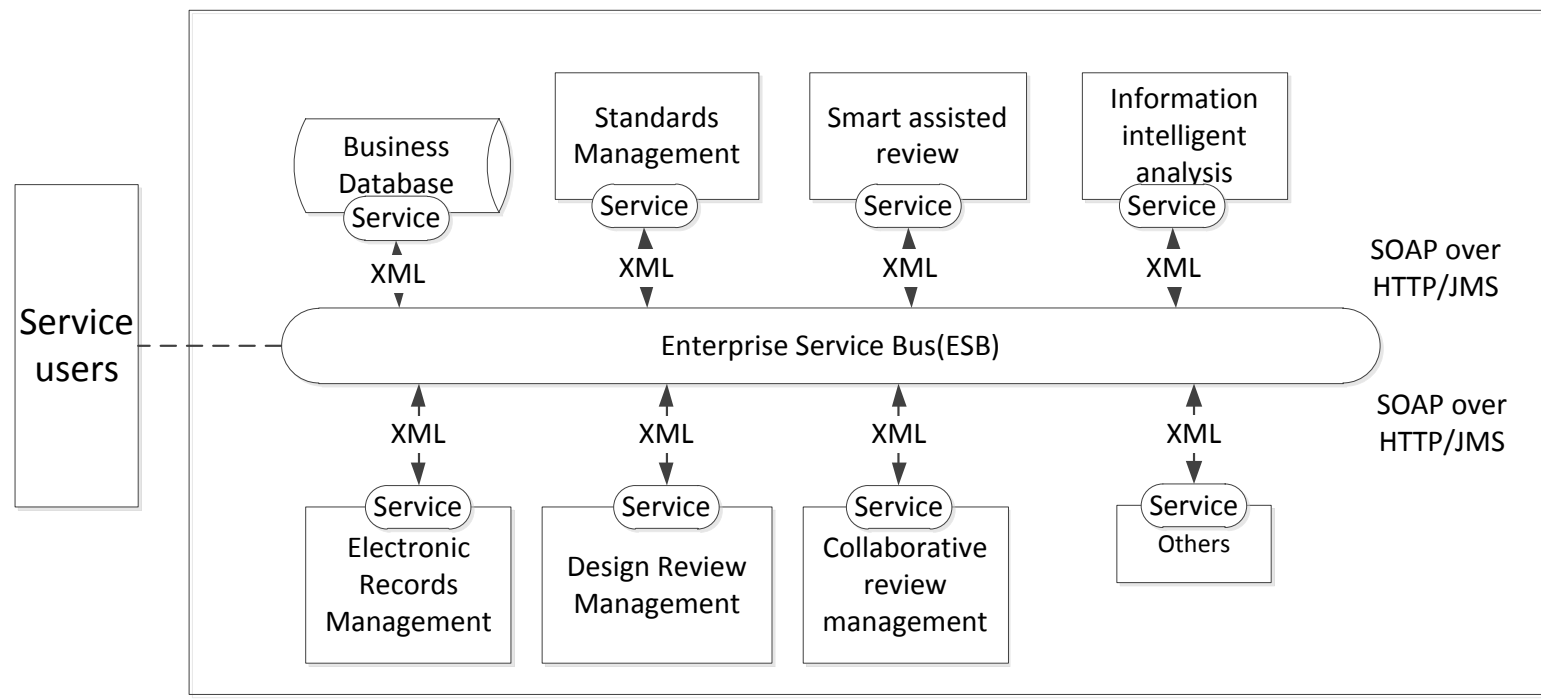

Figure 1. SOA service application model

When enterprise uses SOA to integrate service platform, the system provides related services, the services and the metadata of services are published to the service directory. System users retrieve the available services from the service directory, transferring the information. In order to call the service, the service users need to pass the platform interface and service interface. In this architecture model, various components are respectively completing the function of each module, having high flexibility and

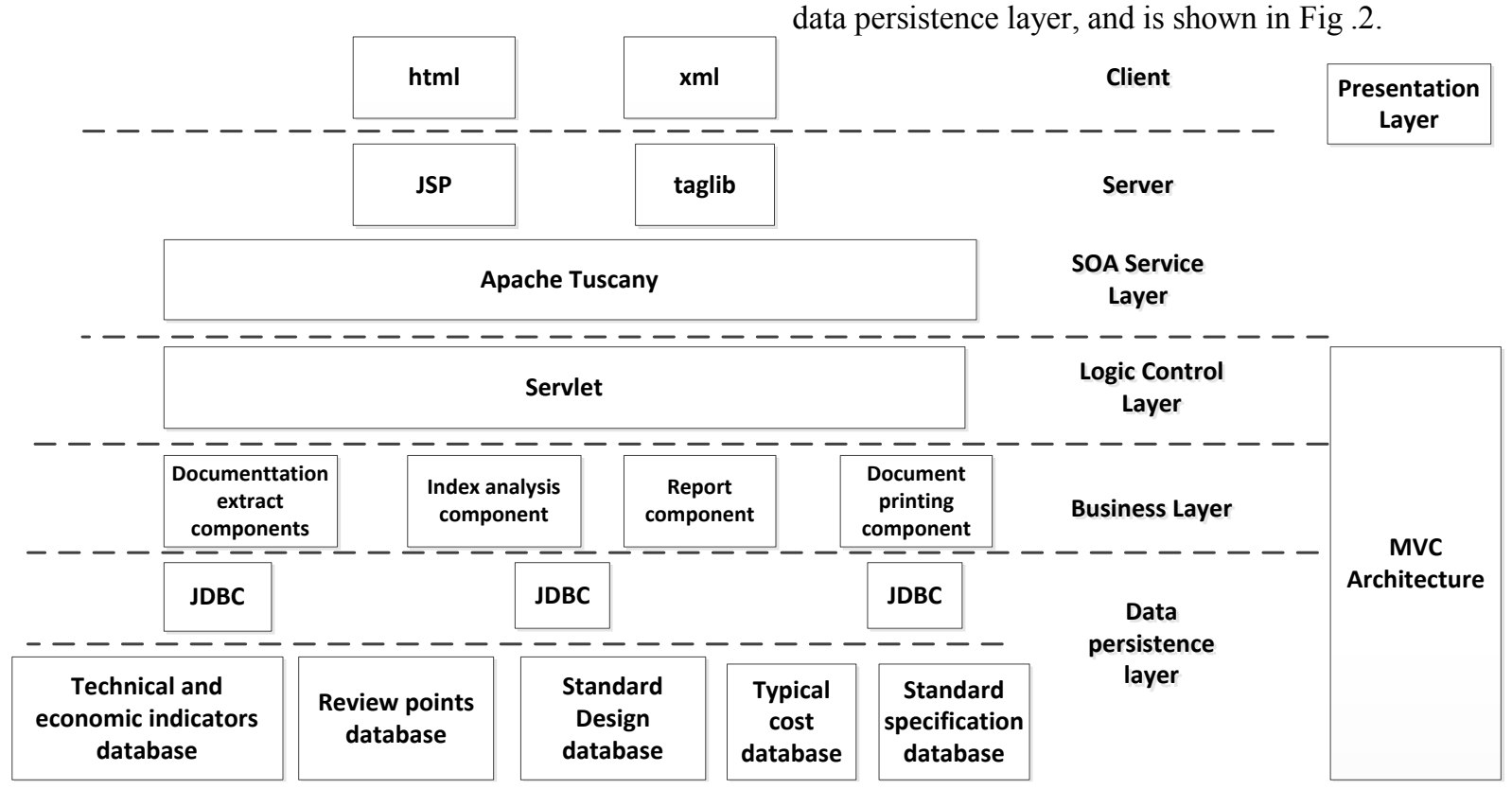

Figure 2. Implementation of SOA service models versatility. The SOA service application model is shown in Fig.1.

\section{B. Implementation of the SOA model based on J2EE}

Implementing the SOA service layer in SSH2 multilayer architecture, ensures the application system can be extended and scaled in performance, and with openness, reusability, extensibility in function. The whole system can be divided into five layers: interface presentation layer, SOA service layer, logic control layer, business layer and data persistence layer, and is shown in Fig .2.

complete the request. The logic control layer in J2EE architecture works on the application server, the users operation is coordinated and the background service call sequence is controlled in this layer. Business function layer consists of packaged function modules, these function modules work together to complete the functions of the system. Data persistence layer is responsible for the 
preservation and management of the data that needs to be persistent stored.

\section{IMPLEMENTATION OF THE SYSTEM MAIN FUNCTIONS}

\section{A. Engineering design files automatically review}

Firstly, build basic databases of different voltage levels and different project types, including technical indicators database, review points database, standard design and typical cost database. When reading the project design documents, fuzzy technical and one of the soft matching algorithm called BPR algorithm are applied to complete vector processing of the unstructured engineering reports, extract the characteristics of the engineering and output review comments, quality score, assessment report automatically. The specific implementation of the flow chart shown in Fig .3.

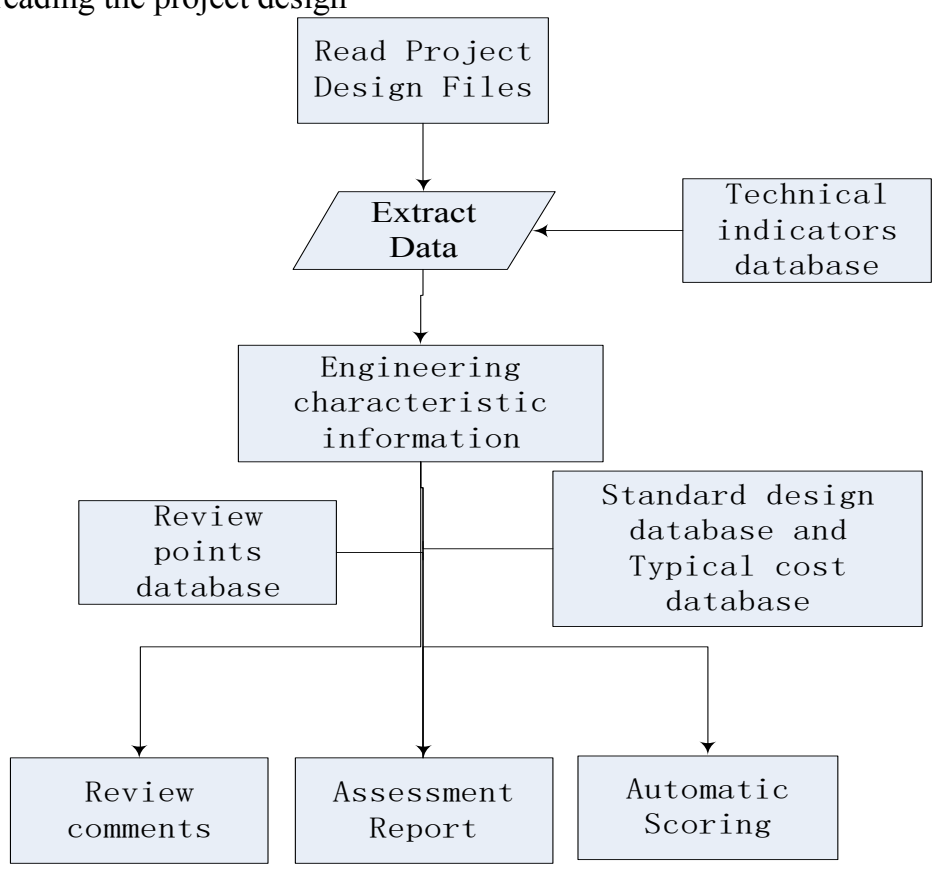

Figure 3. Flow chart of automatic review

\section{B. Collaborative evaluation}

When reviewing the designs in traditional way, experts need to fill in Word, Excel, and other electronic documents firstly, and all review comments and major issue tables are collected by artificial means. The collaborative assessment module of the intelligent design review management system can help experts fill in the review comments quickly and efficiently, and automatically generate major issues statistics tables, and technical respond tables. Specific user interface shown if Fig .4.

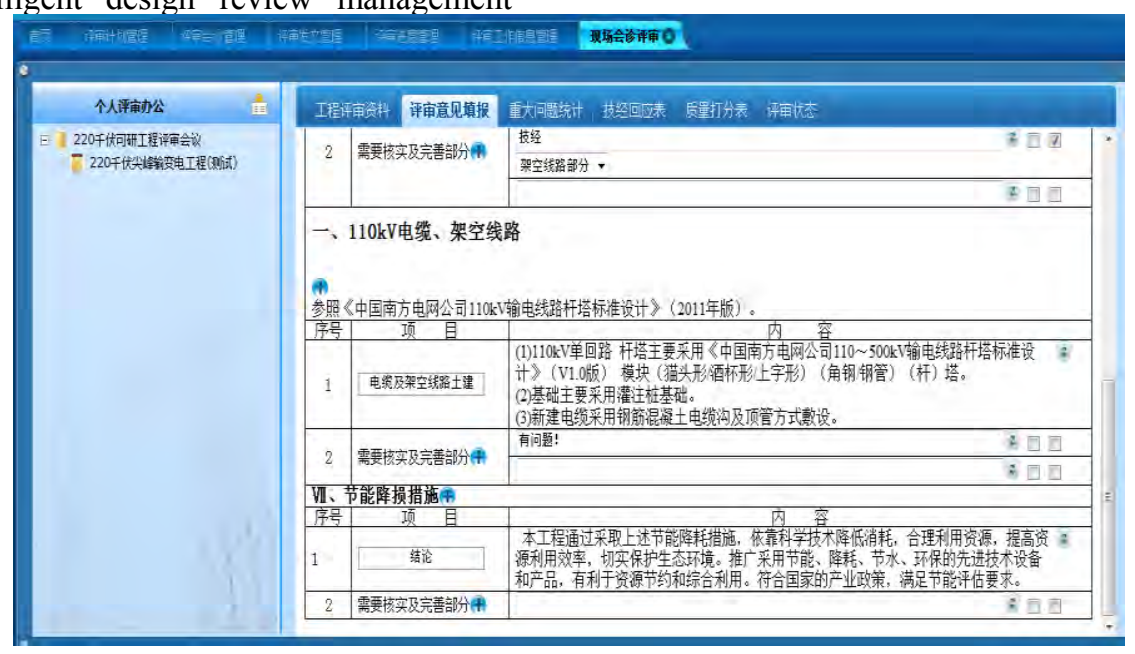

Figure 4. Collaborative evaluation operation interface 


\section{Multi-dimensional analysis of power engineering properties}

Establish power transmission project construction cost database based on the project design files and review results, considering the project type, the assessment phase, voltage level, region, time and other dimensions, statistics and analysis the number of construction projects, the scale, the body cost, the cost distribution, the mean cost and other key indicators, and automatically form the related key charts. These charts can directly reflect the key factors and quality defects of the engineering, and generate analysis reports, greatly improving the efficiency of statistical analysis.

The effect of statistical analysis of the project construction is shown in Fig .5. And Project cost statistical results shown in Fig .6.

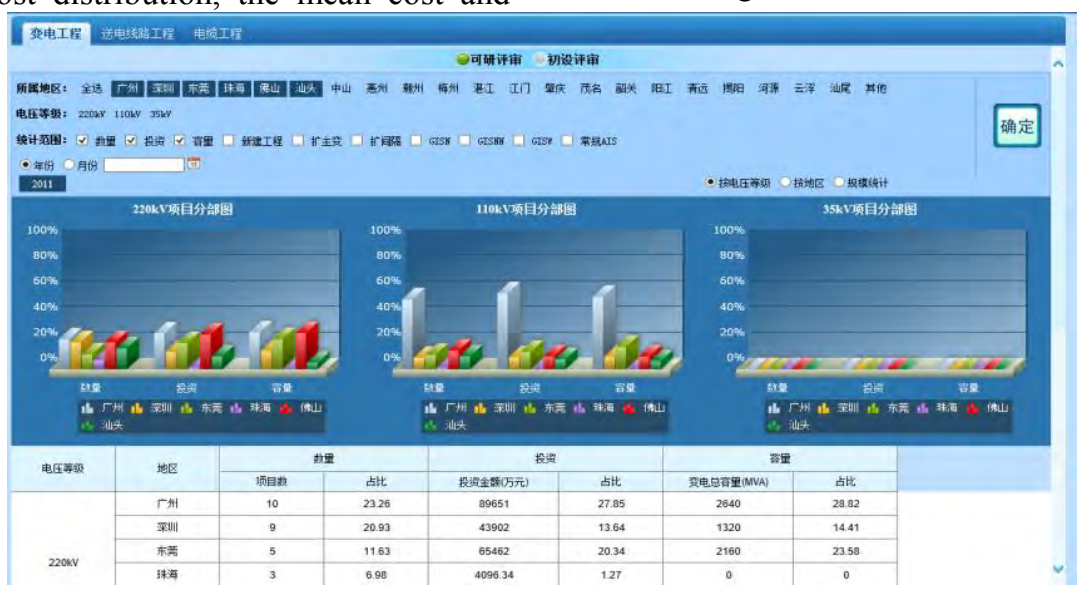

Figure 5. The project construction display interface

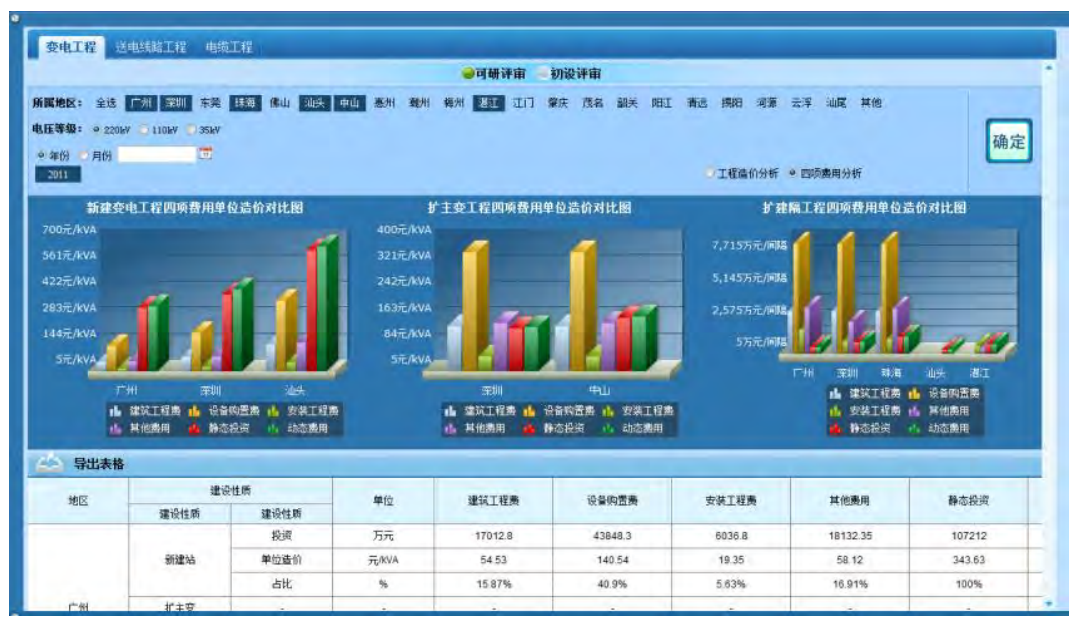

Figure 6. Project cost display interface

\section{CONCLUSion}

Intelligent design review system of power transmission projects is based on the current mainstream enterprise software development technology, text retrieval and extraction technology, and large database technology, built an information support system that integrated assessment, management, information and communication. Thus the review process is cured, the assessment standards are uniform, and the plan execution is strengthened. The application of the system can effectively accelerate the process of review and approval, can improve the quality and efficiency of the project review, meanwhile the operating costs is reduced and the operational efficiency is enhanced. The system has been successfully put into operation in Guangdong Power Grid Corporation planning assessment center, and has brought great social and economic benefits for the corporation. The achievements have great popularization and application value.

\section{REFERENCES}

[1] LIU Ning, ZHU Wei-wei, and WEI Xiang, "Application of SOA to Information Integration of Electric Power Enterprises," Electric Power ICT,vol. 11, Oct. 2013, pp.109-112.

[2] WANG Xiaohui,'Research on Key Technologies of Electric Power GIS Based on SOA [D],'Notrh China Electric Power University,2012

[3] LIU Guo-min, SONG Yu, TENG Xiao-lei, and ZHOU Qingjie," Study power information integration platform based on SOA," Electric Power,vol. 45,Jun. 2012,pp.96-99.

[4] Hong Yingdi,"Design and implementation of Fujian power transmission project design review system [D]," University of Electronic Science and Technology of China,2013

[5] LIU Youcai,'Design and Evaluation of Intelligent Power Grid in Harbin [D]," North China Electric Power University,2012 
[6] ZHOU Yuncheng, XU Tongyu, and FU Lisi," Electric Power GIS System Architecture Based on CIM and SOA," Power System Technology,vol. 38,Apr. 2014,pp.1115-1120.

[7] LIU Jingyi,"Design and implement of SOA-based framework for Bank Management Information System [D]," Shanghai Jiaotong University,2009
[8] KANG Xiaojun,"Research on Evaluation of Professional Title Based on Service-oriented Architecture," Science and Technology Management Research,Feb.2011,pp.107-111.

[9] DONG Denghui,"A SOA Reference Model Based on Multigranularity Service[D]," Zhejiang University of Technology,2012

[10] CUI Jianlei,"Research and Implementation of SOA-based Power System Security Risk Assessment System[D],"Zhejiang University,2013 